DESY 98-062

$\mathrm{MZ}-\mathrm{TH} / 98-21$

hep-th/9805215

\title{
The Complete Solution of the Classical $\mathrm{SL}(2, \mathbb{R}) / \mathrm{U}(1)$ Gauged WZNW Field Theory
}

\author{
Uwe Müller $^{1}$, Gerhard Weigt ${ }^{2}$ \\ ${ }^{1}$ Institut für Physik, Johannes-Gutenberg-Universität Mainz \\ Staudingerweg 7, D-55099 Mainz, Germany \\ ${ }^{2}$ Deutsches Elektronen-Synchrotron DESY Zeuthen \\ Platanenallee 6, D-15738 Zeuthen, Germany \\ E-mail: umueller@thep.physik.uni-mainz.de, weigt@ifh.de
}

\begin{abstract}
We prove that any gauged WZNW model has a Lax pair representation, and give explicitly the general solution of the classical equations of motion of the $\mathrm{SL}(2, \mathbb{R}) / \mathrm{U}(1)$ theory. We calculate the symplectic structure of this solution by solving a differential equation of the Gelfand-Dikii type with initial state conditions at infinity, and transform the canonical physical fields non-locally onto canonical free fields. The results will, finally, be collected in a local Bäcklund transformation. These calculations prepare the theory for an exact canonical quantization.
\end{abstract}

God does not care about our mathematical difficulties. He integrates empirically.

Albert Einstein

\section{Introduction}

To get space-time structure from conformal Wess-Zumino-Novikov-Witten (WZNW) theories is an especially tempting but mathematically non-trivial problem. The standard procedure integrates out functionally the gauge field of a gauged WZNW model and attempts a sigma-model interpretation of the arising effective action. An outstanding example is the $\operatorname{SL}(2, \mathbb{R}) / \mathrm{U}(1)$ coset theory which gives a space-time black hole metric [1]. However, that 
procedure neither preserves conformal invariance in a convincing manner 11 , 2, 34 nor delivers an exact effective action [4 for dynamical calculations. The path-integration left uncalculated a functional determinant of a differential operator [5] which might influence previous results.

Therefore, it would be desirable to have a mathematically well-defined method. In this paper we take up again the discussion of the $\operatorname{SL}(2, \mathbb{R}) / \mathrm{U}(1)$ gauged WZNW theory and follow an entirely classical approach based on an exact effective action [6] obtained by eliminating the non-dynamical U(1) gauge field algebraically instead of path-integrating it out. The arising coset theory is conformal and integrable [7]. More generally, we prove that integrability holds for any gauged WZNW theory by deriving a Lax pair for its equation of motion. So far this was known only for nilpotent gauging which yields Toda theories [8, 9]. We do not look here for algebraic coset current constructions [10] but present the general analytic solution of the classical equations of motion of the $\mathrm{SL}(2, \mathbb{R}) / \mathrm{U}(1)$ theory, and we derive the symplectic structure for a field-theoretic situation by solving a Gelfand-Dikii type second order differential equation with boundary conditions at infinity. The likewise solved problem with periodic boundary conditions is more involved due to zero modes. This solution which describes a closed string moving in the background of a black hole target-space metric will be prepared for canonical quantization in a separate paper. Canonical quantization of the $\mathrm{SL}(2, \mathbb{R}) / \mathrm{U}(1)$ model should be possible once we have derived from the symplectic structure canonical transformations of the physical fields onto free fields. These transformations are non-local, but this non-locality can be hidden superficially in a local Bäcklund transformation [7].

Since all considerations are classical there will be no dilaton here which usually arises in pertubative $\beta$-function calculations due to the curved targetspace metric of the model [11]. We hope that quantization based on the given exact analytical solution will help to clarify some of the discussions [1, 2, 3] related to a possibly non-perturbative dilaton. Black hole radiation could also appear in a new light once we have quantized the theory. However, it seems to be reasonable to consider this Lagrangean coset model in its own right as an integrable conformal field theory with space-time structure. First results were published in ref. [7].

We begin our calculations by first proving that any $G / H$ gauged WZNW theory is integrable. It will be shown that the non-linear equations of motion can be expressed by a linear Lax pair representation. 


\section{The Integrability of the G/H Gauged WZNW Theory}

Wess-Zumino-Novikov-Witten models [12 are $\sigma$-models

$$
S_{\mathrm{WZNW}}[g]=\frac{k}{8 \pi} \int_{M} \gamma^{\mu \nu} \operatorname{tr}\left(g^{-1} \partial_{\mu} g g^{-1} \partial_{\nu} g\right) \sqrt{-\gamma} \mathrm{d}^{2} \xi+k I_{\mathrm{WZ}}[g]
$$

which include the additional topological Wess-Zumino term

$$
I_{\mathrm{WZ}}=\frac{1}{12 \pi} \int_{B, \partial B=M} \operatorname{tr}\left(g^{-1} \mathrm{~d} g \wedge g^{-1} \mathrm{~d} g \wedge g^{-1} \mathrm{~d} g\right)
$$

integrated over a volume $B$ with the boundary $\partial B=M$. They describe conformal and integrable theories which are globally invariant under left and right multiplications with elements of the symmetry group $G$

$$
S_{\mathrm{WZNW}}[g]=S_{\mathrm{WZNW}}\left[a^{-1} g b\right], \quad a, b \in G, \quad a, b=\text { const. }
$$

The field $g(\tau, \sigma)$ takes values in the group $G, \gamma_{\mu \nu}$ is the Minkowskian metric of the world surface $M$ parametrized by $\xi=\{\tau, \sigma\}, \gamma$ its determinant, and $k$ is the coupling parameter of the theory.

In order to get from (11) the $G / H$ gauged action, we have to embed the subgroup $H$ of $G$ into the symmetry group $G_{\mathrm{L}} \times G_{\mathrm{R}}$ of left and right multiplications by homomorphisms

$$
\begin{array}{ll}
L: H \rightarrow G_{\mathrm{L}}, & h \mapsto L(h), \\
R: H \rightarrow G_{\mathrm{R}}, & h \mapsto R(h) .
\end{array}
$$

Then the gauge transformations corresponding to (3) become

$$
g \rightarrow L\left(h^{-1}\right) g R(h), \quad h \in H .
$$

The homomorphic mappings $L$ and $R$ generate linear mappings $L^{\prime}$ und $R^{\prime}$ of the Lie algebra of $H$ into the Lie algebra of $G$

$$
\begin{aligned}
& L^{\prime}: \operatorname{Lie}(H) \rightarrow \operatorname{Lie}(G), \quad T^{i} \mapsto L^{\prime}\left(T^{i}\right)=\left.\frac{\mathrm{d}}{\mathrm{d} t} L\left(\exp \left(T^{i} t\right)\right)\right|_{t=0}, \\
& R^{\prime}: \operatorname{Lie}(H) \rightarrow \operatorname{Lie}(G), \quad T^{i} \mapsto R^{\prime}\left(T^{i}\right)=\left.\frac{\mathrm{d}}{\mathrm{d} t} R\left(\exp \left(T^{i} t\right)\right)\right|_{t=0},
\end{aligned}
$$


where $T^{i}$ are the elements of the Lie algebra of $H$. Then the infinitesimal gauge transformations of (5) become

$$
\delta g=g R^{\prime}(\delta h)-L^{\prime}(\delta h) g .
$$

Usually the invariance under local gauge transformations $\delta h(\tau, \sigma)$ is found by introducing an $H$ gauge field $A_{\mu}$ with the transformation properties

$$
A_{\mu} \rightarrow h^{-1} A_{\mu} h-h^{-1} \partial_{\mu} h
$$

or infinitesimally

$$
\delta A_{\mu}=-\partial_{\mu} \delta h+\left[A_{\mu}, \delta h\right],
$$

and replacing partial derivatives by covariant ones

$$
\partial_{\mu} g \rightarrow D_{\mu} g \equiv \partial_{\mu} g-L^{\prime}\left(A_{\mu}\right) g+g R^{\prime}\left(A_{\mu}\right)
$$

But this does not guarantee the local gauge invariance of the WZ term (2) unless the anomaly cancellation condition

$$
\operatorname{tr}\left(L^{\prime} \otimes L^{\prime}-R^{\prime} \otimes R^{\prime}\right)=0
$$

is fulfilled. One could specialize this condition to a nilpotent gauge

$$
L^{\prime} \otimes L^{\prime}=0, \quad R^{\prime} \otimes R^{\prime}=0,
$$

which reduces WZNW models to Toda theories. One could also choose a vector gauge

$$
L(h)=R(h) \quad \Rightarrow \quad L^{\prime}=R^{\prime}
$$

or for abelian gauge groups the axial gauge

$$
L(h)=R\left(h^{-1}\right) \quad \Rightarrow \quad L^{\prime}=-R^{\prime} .
$$

Without any specialization, after some manipulations, the $G / H$ gauged WZNW model written in light cone coordinates $z=\tau+\sigma, \bar{z}=\tau-\sigma$ becomes

$$
\begin{aligned}
S_{\text {WZNW, gauged }}[g, A]= & S_{\text {WZNW }}[g]+\frac{k}{2 \pi} \int_{M} \mathrm{~d} z \mathrm{~d} \bar{z} \times \\
\times & {\left[\operatorname{tr}\left(R^{\prime}\left(A_{z}\right) g^{-1} \partial_{\bar{z}} g\right)-\operatorname{tr}\left(L^{\prime}\left(A_{\bar{z}}\right) \partial_{z} g g^{-1}\right)-\right.} \\
& \left.\quad-\operatorname{tr}\left(R^{\prime}\left(A_{z}\right) g^{-1} L^{\prime}\left(A_{\bar{z}}\right) g\right)+\operatorname{tr}\left(R^{\prime}\left(A_{z}\right) R^{\prime}\left(A_{\bar{z}}\right)\right)\right] .
\end{aligned}
$$


The local gauge invariance of this action remains valid if the gauge field $A_{\mu}$, which behaves non-dynamically here and is, therefore, purely algebraically determined by its field equation, is eliminated by this equation. Then the equation of motion for the field $g(\tau, \sigma)$ follows by varying this action with respect to $g$ only. The variation of $A_{\mu}$ as a function of $g$ does not contribute due to the extremal pinciple. With the definitions

$$
B \equiv R^{\prime}\left(A_{z}\right), \quad \bar{B} \equiv g^{-1} L^{\prime}\left(A_{\bar{z}}\right) g
$$

the equation of motion of $g(\tau, \sigma)$ becomes

$$
\partial_{z}\left(g^{-1} \partial_{\bar{z}} g\right)-\left[B, g^{-1} \partial_{\bar{z}} g\right]-\partial_{z} \bar{B}+\partial_{\bar{z}} B+[B, \bar{B}]=0 .
$$

These equations can be rewritten as a flatness condition

$$
\left[\partial_{z}-B, g^{-1} \partial_{\bar{z}} g\right]+\left[\partial_{z}-B, \partial_{\bar{z}}-\bar{B}\right]=\left[\partial_{z}-B, \partial_{\bar{z}}+g^{-1} \partial_{\bar{z}} g-\bar{B}\right]=0,
$$

which is the Lax pair representation we are looking for. The commutativity of the linear Lax operators $\left(\partial_{z}-B\right)$ and $\left(\partial_{\bar{z}}+g^{-1} \partial_{\bar{z}} g-\bar{B}\right)$ is the necessary condition in order that the system of linear first order differential equations

$$
\left(\partial_{z}-B\right) \Psi=0, \quad\left(\partial_{\bar{z}}+g^{-1} \partial_{\bar{z}} g-\bar{B}\right) \Psi=0
$$

has a non-trivial solution. This proves the integrability of $G / H$ gauged WZNW models.

We should mention here that the equation of motion (17) is an equation between elements of the Lie algebra $G$. It describes in components $\operatorname{dim} G$ equations for $\operatorname{dim} G-\operatorname{dim} H$ fields. This becomes manifest by gauge transforming the gauge covariant form of (17)

$$
D_{z}\left(g^{-1} D_{\bar{z}} g\right)-R^{\prime}\left(F_{z \bar{z}}\right)=0
$$

into

$$
R\left(h^{-1}\right)\left(D_{z}\left(g^{-1} D_{\bar{z}} g\right)-R^{\prime}\left(F_{z \bar{z}}\right)\right) R(h)=0,
$$

where

$$
F_{\mu \nu} \equiv \partial_{\mu} A_{\nu}-\partial_{\nu} A_{\mu}-\left[A_{\mu}, A_{\nu}\right]
$$

is the field strength with the gauge transformations

$$
F_{\mu \nu} \rightarrow h^{-1} F_{\mu \nu} h
$$

Parametrizing the group elements $g$ analogously to a Gaußian decomposition

$$
\begin{aligned}
& g\left(x^{\alpha}, y^{a}\right)=L\left(h\left(y^{a}\right)\right)^{-1} g_{0}\left(x^{\alpha}\right) R\left(h\left(y^{a}\right)\right), \quad \alpha=1, \ldots, \operatorname{dim} G-\operatorname{dim} H, \\
& a=1, \ldots, \operatorname{dim} H \text {, }
\end{aligned}
$$


the equations of motions for the fields $x^{\alpha}, y^{a}$ take the form

$$
R\left(h\left(y^{a}\right)\right)^{-1}\left(D_{z}\left(g_{0}\left(x^{\alpha}\right)^{-1} D_{\bar{z}} g_{0}\left(x^{\alpha}\right)\right)-R^{\prime}\left(F_{z \bar{z}}\left[g_{0}\left(x^{\alpha}\right)\right]\right)\right) R\left(h\left(y^{a}\right)\right)=0 .
$$

Indeed, due to gauge invariance we have equations of motion for the fields $x^{\alpha}$ only. But we can also derive the $\operatorname{dim} H$ identities among the $\operatorname{dim} G$ components of the equation of motion by varying the action (15) with respect to the pure gauge transformations (7), and using the decomposition

$$
A_{z}=A_{z}^{a} H^{a}, \quad A_{\bar{z}}=A_{\bar{z}}^{a} H^{a}
$$

as well as the mapping (6) in the form

$$
R^{\prime}\left(H^{a}\right)=R^{a}, \quad L^{\prime}\left(H^{a}\right)=L^{a}, \quad R^{a}, L^{a} \in \operatorname{Lie}(G) .
$$

Thus there are $\operatorname{dim} H$ identities

$$
\operatorname{tr}\left(\left(D_{z}\left(g^{-1} D_{\bar{z}} g\right)-R^{\prime}\left(F_{z \bar{z}}\right)\right)\left(R^{a}-g^{-1} L^{a} g\right)\right)=0 .
$$

The Lax pair representation (18) is likewise overdetermined, and it can be reduced as well by equations (28) to an independent set of conditions.

In the following we shall restrict ourselves exclusively to the $\mathrm{SL}(2, \mathbb{R}) / \mathrm{U}(1)$ gauged WZNW theory.

\section{The Classical Effective Action of the $\mathrm{SL}(2, \mathbb{R}) / \mathrm{U}(1)$ Gauged WZNW Theory}

We parametrize the group $\operatorname{SL}(2, \mathbb{R})$ in accordance with $(24)$ by

$$
\begin{aligned}
g= & g(r, t, \alpha)=\exp ((2 \alpha-t) I / 2) \exp (r J) \exp ((2 \alpha+t) I / 2) \\
= & \left(\begin{array}{cc}
\cosh r \cos 2 \alpha+\sinh r \cos t \quad \cosh r \sin 2 \alpha+\sinh r \sin t \\
-\cosh r \sin 2 \alpha+\sinh r \sin t \quad \cosh r \cos 2 \alpha-\sinh r \cos t
\end{array}\right), \\
& 0 \leq r<\infty, \quad 0 \leq t<2 \pi, \quad 0 \leq \alpha<\pi,
\end{aligned}
$$

where the Lie algebra elements $I$ and $J$ are the matrices

$$
I=\left(\begin{array}{rr}
0 & 1 \\
-1 & 0
\end{array}\right), \quad J=\left(\begin{array}{rr}
1 & 0 \\
0 & -1
\end{array}\right)
$$

and gauge the subgroup $U(1)$ with group elements $h$ and generator i

$$
h(\alpha)=\exp (\mathrm{i} \alpha), \quad \alpha \in \mathbb{R}
$$


according to the recipe (丑), (6)

$$
L, R: H \rightarrow G, \quad h=\exp (\mathrm{i} \alpha) \mapsto L(h)=\exp (-\alpha I), R(h)=\exp (\alpha I)
$$

in the axial gauge

$$
-L^{1}=-L^{\prime}\left(H^{1}\right)=R^{1}=R^{\prime}\left(H^{1}\right)=I .
$$

Then (15) becomes the $\mathrm{SL}(2, \mathbb{R}) / \mathrm{U}(1)$ gauged WZNW action

$$
\begin{aligned}
& S_{\text {WZNW,gauged }}[r, t, \alpha, A]=\frac{k}{2 \pi} \int_{M} \mathrm{~d} z \mathrm{~d} \bar{z}\left(\partial_{z} r \partial_{\bar{z}} r+\tanh ^{2} r \partial_{z} t \partial_{\bar{z}} t-\right. \\
& \left.-4 \cosh ^{2} r\left(A_{z}^{1}+\partial_{z} \alpha+\frac{1}{2} \tanh ^{2} r \partial_{z} t\right)\left(A_{\bar{z}}^{1}+\partial_{\bar{z}} \alpha-\frac{1}{2} \tanh ^{2} r \partial_{\bar{z}} t\right)\right)
\end{aligned}
$$

which can be considered as a Lagrangean formulation of the algebraic coset current construction [10], in case, we make use of the gauge field $A_{\mu}$ as a Lagrangean multiplier. This is possible because, as was mentioned before, the gauge field is non-dynamical. It is purely algebraically determined by the field equations

$$
A_{z}^{1}=-\partial_{z} \alpha-\frac{1}{2} \tanh ^{2} r \partial_{z} t \quad \text { and } \quad A_{\bar{z}}^{1}=-\partial_{\bar{z}} \alpha+\frac{1}{2} \tanh ^{2} r \partial_{\bar{z}} t
$$

which imply in connection with the equations of motions of the fields $r, t$ a vanishing field strength [6]

$$
F_{z \bar{z}}^{1}=\partial_{z} A_{\bar{z}}^{1}-\partial_{\bar{z}} A_{z}^{1}=0
$$

Since the field $\alpha$ of (29) behaves in accordance with (25) as well non-dynamically, we choose the gauge $\alpha=0$.

These considerations allow us to define, entirely classically, the effective action of the $\mathrm{SL}(2, \mathbb{R}) / \mathrm{U}(1)$ gauged WZNW theory in a gauge invariant manner by eliminating the gauge field through the algebraic equation (35) [6]. The result

$$
S_{\text {WZNW,gauged }}[r, t]=\frac{k}{2 \pi} \int_{M} \mathrm{~d} z \mathrm{~d} \bar{z}\left(\partial_{z} r \partial_{\bar{z}} r+\tanh ^{2} r \partial_{z} t \partial_{\bar{z}} t\right)
$$

is conformal and it describes an integrable theory. It yields, as we know, the same equations of motion for the fields $r, t$ as the action (34). We could also get this action by formally Gauß-integrating over the gauge field and while 
doing so neglecting possible anomalies. The more reasonable path-integration over the gauge field decomposed in terms of scalar fields $\phi, \chi$

$$
A_{\mu}=\partial_{\mu} \phi+\epsilon_{\mu}{ }^{\nu} \partial_{\nu} \chi
$$

yields quantum contributions to the effective action, but it is incomplete as well [4] because the functional determinant $\operatorname{Det}(M)$ of the operator [5]

$$
M=-\left(\begin{array}{cc}
\partial^{2}+\partial^{\mu} g \partial_{\mu} & \epsilon^{\mu \nu} \partial_{\mu} g \partial_{\nu} \\
-\epsilon^{\mu \nu} \partial_{\mu} g \partial_{\nu} & \partial^{2}+\partial^{\mu} g \partial_{\mu}
\end{array}\right), \quad g=\ln \cosh ^{2} r+\ln \frac{k}{\pi}
$$

resists exact calculation so far.

The classical action (37) is, therefore, a reliable basis for an exact discussion of the $\mathrm{SL}(2, \mathbb{R}) / \mathrm{U}(1)$ theory, and we hope that it is a useful one for quantization. It also has a nice $\sigma$-model interpretation [1]: the target-space metric

$$
\mathrm{d} s^{2}=\mathrm{d} r^{2}+\tanh ^{2} r \mathrm{~d} t^{2}
$$

shows in light cone coordinates

$$
u=-\sinh r \mathrm{e}^{\mathrm{i} t}, \quad \bar{u}=\sinh r \mathrm{e}^{-\mathrm{i} t}
$$

after Wick rotation $t \rightarrow$ it a two-dimensional black hole singularity

$$
\mathrm{d} s^{2}=-\frac{\mathrm{d} u \mathrm{~d} \bar{u}}{1-u \bar{u}}
$$

with singular curvature tensor. And as a conformal theory the action describes a string moving in the background of a black hole.

\section{Equations of Motion, Conservation Laws and the Gelfand-Dikii Equation}

The equations of motion of the action (37) (or (34))

$$
\begin{aligned}
\partial_{z} \partial_{\bar{z}} r & =\frac{\sinh r}{\cosh ^{3} r} \partial_{z} t \partial_{\bar{z}} t \\
\partial_{z} \partial_{\bar{z}} t & =-\frac{1}{\sinh r \cosh r}\left(\partial_{z} r \partial_{\bar{z}} t+\partial_{z} t \partial_{\bar{z}} r\right) .
\end{aligned}
$$

guarantee conservation of the chiral component of the energy-momentum tensor (we will not indicate, whenever possible, the similar anti-chiral parts) $(\gamma=\sqrt{2 \pi / k})$

$$
T \equiv T_{z z}=\frac{1}{\gamma^{2}}\left(\left(\partial_{z} r\right)^{2}+\tanh ^{2} r\left(\partial_{z} t\right)^{2}\right)
$$


and vanishing trace

$$
T_{z \bar{z}}+T_{\bar{z} z}=0
$$

shows the conformal invariance of the theory.

Moreover, by multiplying currents of the ungauged SL $(2, \mathbb{R}) / \mathrm{U}(1)$ WZNW model with Wilson-line factors [6] there arise two further conserved chiral quantities on shell

$$
V_{ \pm}=\frac{1}{\gamma^{2}} \mathrm{e}^{ \pm \mathrm{i} \nu}\left(\partial_{z} r \pm \mathrm{i} \tanh r \partial_{z} t\right),
$$

if $\nu$ satisfies

$$
\partial_{z} \nu=\left(1+\tanh ^{2} r\right) \partial_{z} t, \quad \partial_{\bar{z}} \nu=\cosh ^{-2} r \partial_{\bar{z}} t .
$$

Since the integrability conditions of these equations just yield the second equation of (43), it will be easy to integrate them, once we have got the general solution of the equations of motion.

Surprisingly, as in the ungauged theory, the energy-momentum tensor has a Sugawara form

$$
T=\gamma^{2} V_{+} V_{-},
$$

although the conformal spin-one quantities $V_{ \pm}$are no usual Kac-Moody currents.

After gauging there remains the local symmetry of the ungauged WZNW action

$$
t \rightarrow t+\delta \varepsilon
$$

with non-chiral Noether currents

$$
J_{z}=\frac{1}{\gamma^{2}} \tanh ^{2} r \partial_{z} t, \quad J_{\bar{z}}=\frac{1}{\gamma^{2}} \tanh ^{2} r \partial_{\bar{z}} t .
$$

But the continuity equation

$$
\partial_{\bar{z}} J_{z}+\partial_{z} J_{\bar{z}}=0
$$

is equivalent, again, to the second of the equations of motion of (43).

For the calculation of the symplectic structure a differential equation of the Gelfand-Dikii type

$$
y^{\prime \prime}-\left(\partial_{z} V_{-} / V_{-}\right) y^{\prime}-\gamma^{2} T y=0
$$

becomes important. This follows from the conserved quantities (44, 46), and we shall show that their solutions will usefully parametrize the general solution of the equtions of motion (43). 


\section{The General Solutions of the Equations of Motion and Gelfand-Dikii Equations}

The general solution of the equations of motion (43) is given by [7]

$$
\begin{aligned}
\sinh ^{2} r & =X \bar{X}, \\
t & =\mathrm{i}(B-\bar{B})+\frac{\mathrm{i}}{2} \ln \frac{X}{\bar{X}},
\end{aligned}
$$

where

$$
\begin{aligned}
& X=A+\frac{\bar{B}^{\prime}}{\bar{A}^{\prime}}(1+A \bar{A}), \\
& \bar{X}=\bar{A}+\frac{B^{\prime}}{A^{\prime}}(1+A \bar{A}),
\end{aligned}
$$

and it is parametrized as in other non-linear theories by arbitrary chiral (anti-chiral) functions $A(z), B(z)(\bar{A}(\bar{z}), \bar{B}(\bar{z}))$ and their derivatives $A^{\prime}(z)$, $B^{\prime}(z)\left(\bar{A}^{\prime}(\bar{z}), \bar{B}^{\prime}(\bar{z})\right)$, respectively. This solution is invariant under internal $\mathrm{GL}(2, \mathbb{C})$ transformations

$$
\begin{aligned}
A \rightarrow T[A] & =\frac{a A-b}{c A+d}, \\
B \rightarrow T[B] & =B+\ln (c A+d), \\
\bar{A} \rightarrow T[\bar{A}] & =\frac{d \bar{A}-c}{b \bar{A}+a}, \\
\bar{B} \rightarrow T[\bar{B}] & =\bar{B}+\ln (b \bar{A}+a), \\
& \left(\begin{array}{rr}
a & -b \\
c & d
\end{array}\right) \in \operatorname{GL}(2, \mathbb{C}) .
\end{aligned}
$$

But it does not factorize into a chiral and anti-chiral part as for ungauged WZNW theories. This solution allows us to integrate the equation for $\nu$ (47)

$$
\nu=t+\mathrm{i}(B+\bar{B})+\mathrm{i} \ln (1+A \bar{A})-\frac{\mathrm{i}}{2} \ln (1+X \bar{X})+\nu_{0},
$$

and the $\mathrm{GL}(2, \mathbb{C})$ invariance is respected if the integration constant $\nu_{0}$ transforms as

$$
\nu_{0} \rightarrow \nu_{0}-\mathrm{i} \ln (a d+b c) .
$$

It is worth mentioning here that this general solution is asymptotically related [13] to the solution of a non-abelian Toda theory [9, 14] which arises by nilpotent gauging a corresponding WZNW model. 
Now it is easy to solve the Gelfand-Dikii equation (52). We rewrite their coefficients using the general solution (53, 54) as

$$
T=\frac{1}{\gamma^{2}}\left(B^{\prime \prime}-B^{\prime 2}-\frac{A^{\prime \prime}}{A^{\prime}} B^{\prime}\right)
$$

and

$$
V_{+}=\frac{1}{\gamma^{2}}\left(\frac{B^{\prime \prime}}{A^{\prime}}-\frac{B^{\prime 2}}{A^{\prime}}-\frac{A^{\prime \prime} B^{\prime}}{A^{\prime 2}}\right) \mathrm{e}^{\mathrm{i} \nu_{0}-2 B}, \quad V_{-}=\frac{1}{\gamma^{2}} A^{\prime} \mathrm{e}^{-\mathrm{i} \nu_{0}+2 B},
$$

and find for the Gelfand-Dikii eqution the two independent solutions

$$
y_{1}=\mathrm{e}^{B}, \quad y_{2}=A \mathrm{e}^{B} .
$$

For the corresponding anti-chiral Gelfand-Dikii equation we get

$$
\bar{y}_{1}=\mathrm{e}^{\bar{B}}, \quad \bar{y}_{2}=\bar{A} \mathrm{e}^{\bar{B}} .
$$

Thus, the general solution of the equations of motion (53, 54) can be reparametrized by the solutions of the Gelfand-Dikii equations $y_{k}(z)$ and $\bar{y}_{k}(\bar{z})$ $(k=1,2)$. A very symmetrical expression arises for the transformed fields $u, \bar{u}(41)$

$$
u=\frac{\bar{y}_{1} y_{1}^{\prime}+\bar{y}_{2} y_{2}^{\prime}}{y_{1} y_{2}^{\prime}-y_{1}^{\prime} y_{2}}, \quad \bar{u}=\frac{y_{1} \bar{y}_{1}^{\prime}+y_{2} \bar{y}_{2}^{\prime}}{\bar{y}_{1} \bar{y}_{2}^{\prime}-\bar{y}_{1}^{\prime} \bar{y}_{2}}
$$

where for simplicity we shall restrict ourselves in the following to the regular solutions by assuming that for finite $z, \bar{z}$

$$
y_{1} y_{2}^{\prime}-y_{1}^{\prime} y_{2} \neq 0, \quad \bar{y}_{1} \bar{y}_{2}^{\prime}-\bar{y}_{1}^{\prime} \bar{y}_{2} \neq 0 \text {. }
$$

The GL(2, C) invariance (55) now becomes

$$
\begin{aligned}
\left(\begin{array}{l}
y_{2} \\
y_{1}
\end{array}\right) \rightarrow\left(\begin{array}{cc}
a & -b \\
c & d
\end{array}\right)\left(\begin{array}{l}
y_{2} \\
y_{1}
\end{array}\right), \quad\left(\begin{array}{l}
\bar{y}_{2} \\
\bar{y}_{1}
\end{array}\right) & \rightarrow\left(\begin{array}{cc}
d & -c \\
b & a
\end{array}\right)\left(\begin{array}{l}
\bar{y}_{2} \\
\bar{y}_{1}
\end{array}\right), \\
& \left(\begin{array}{cc}
a & -b \\
c & d
\end{array}\right) \in \operatorname{GL}(2, \mathbb{C}) .
\end{aligned}
$$

These results are useful to find the complete symplectic structure of the theory, which is defined by the canonical Poisson brackets of the physical fields $r(\tau, \sigma), t(\tau, \sigma)$ at equal $\tau$ (writing up here and in the following the non-vanishing brackets only!)

$$
\begin{aligned}
& \left\{r(\tau, \sigma), \dot{r}\left(\tau, \sigma^{\prime}\right)\right\}=\gamma^{2} \delta\left(\sigma-\sigma^{\prime}\right), \quad\left\{t(\tau, \sigma), \dot{t}\left(\tau, \sigma^{\prime}\right)\right\}=\gamma^{2} \operatorname{coth}^{2} r \delta\left(\sigma-\sigma^{\prime}\right), \\
& \left\{\dot{r}(\tau, \sigma), \dot{t}\left(\tau, \sigma^{\prime}\right)\right\}=2 \gamma^{2} \frac{\dot{t}(\sigma, \tau)}{\sinh r(\tau, \sigma) \cosh r(\tau, \sigma)} \delta\left(\sigma-\sigma^{\prime}\right) .
\end{aligned}
$$


According to this the canonical conjugated momenta $\pi_{r}$ and $\pi_{t}$ are

$$
\pi_{r}(\tau, \sigma)=\frac{1}{\gamma^{2}} \dot{r}, \quad \pi_{t}(\tau, \sigma)=\frac{1}{\gamma^{2}} \tanh ^{2} r \dot{t} .
$$

The corresponding Poisson brackets of the $u, \bar{u}$ fields become

$$
\begin{aligned}
& \left\{u(\tau, \sigma), \dot{\bar{u}}\left(\tau, \sigma^{\prime}\right)\right\}=\left\{\bar{u}(\tau, \sigma), \dot{u}\left(\tau, \sigma^{\prime}\right)\right\}=2 \gamma^{2}(1+u \bar{u}) \delta\left(\sigma-\sigma^{\prime}\right), \\
& \left\{\dot{u}(\tau, \sigma), \dot{\bar{u}}\left(\tau, \sigma^{\prime}\right)\right\}=2 \gamma^{2}(\dot{u} \bar{u}-u \dot{\bar{u}}) \delta\left(\sigma-\sigma^{\prime}\right) .
\end{aligned}
$$

It is a non-trivial problem to calculate the different (anti-)chiral functions of the theory in terms of the physical fields. This could be done by solving the Gelfand-Dikii equtions. However, because of the $\operatorname{GL}(2, \mathbb{C})$ invariance these functions are determined up to four complex constants only, and we have to fix the $\mathrm{GL}(2, \mathbb{C})$ invariance in order to be able to solve this initial state problem uniquely.

\section{The Solution of an Initial State Problem}

We assume that the physical fields at the 'time' $\tau_{0}$ take the initial values

$$
r\left(\tau_{0}, \sigma\right)=r_{0}(\sigma), \quad t\left(\tau_{0}, \sigma\right)=t_{0}(\sigma), \quad \dot{r}\left(\tau_{0}, \sigma\right)=r_{1}(\sigma), \quad \dot{t}\left(\tau_{0}, \sigma\right)=t_{1}(\sigma) .
$$

This also fixes the initial state of the $u, \bar{u}$ fields. Then the $A(z), B(z), \bar{A}(\bar{z})$, $\bar{B}(\bar{z})$ are completely determined through the solutions of the Gelfand-Dikii equations $y_{k}(z), \bar{y}_{k}(\bar{z})(60,61)$, in case, the $\mathrm{GL}(2, \mathbb{C})$ invariance (55, 64) is fixed by four additional initial values. But this solves the initial state problem of the two Gelfand-Dikii equations only if we take into consideration that these two chiral respectively anti-chiral second order differential equations are equivalent to the four non-chiral first order differential eqations

$$
\begin{aligned}
y_{1}^{\prime} & =\frac{\partial_{z} \bar{u}}{1+u \bar{u}}\left(u y_{1}-\bar{y}_{2}\right), & y_{2}^{\prime} & =\frac{\partial_{z} \bar{u}}{1+u \bar{u}}\left(u y_{2}+\bar{y}_{1}\right), \\
\bar{y}_{1}^{\prime} & =\frac{\partial_{\bar{z}} u}{1+u \bar{u}}\left(\bar{u} \bar{y}_{1}-y_{2}\right), & \bar{y}_{2}^{\prime} & =\frac{\partial_{\bar{z}} u}{1+u \bar{u}}\left(\bar{u} \bar{y}_{2}+y_{1}\right),
\end{aligned}
$$

which follow from the general solution (62).

We fix here the $\mathrm{GL}(2, \mathbb{C})$ invariance by the asymptotic boundary conditions

$$
\begin{aligned}
& \left.y_{k}\right|_{\sigma=-\infty}=C_{k},\left.\quad \bar{y}_{k}\right|_{\sigma=-\infty}=\bar{C}_{k}, \\
& C_{k}, \bar{C}_{k} \in \mathbb{C}, \quad k \in\{1,2\} .
\end{aligned}
$$


These boundary conditions solve the first order differential equations (69) uniquely. Taking into consideration that the integration of (47) in terms of $r, t$

$$
\nu(\tau, \sigma)=t(\tau, \sigma)+\int_{-\infty}^{\sigma} \mathrm{d} \sigma^{\prime} \tanh ^{2} r\left(\tau, \sigma^{\prime}\right) \dot{t}\left(\tau, \sigma^{\prime}\right)
$$

is defined if

$$
\lim _{\sigma \rightarrow \pm \infty} r(\tau, \sigma)=0, \quad \lim _{\sigma \rightarrow \pm \infty} t(\tau, \sigma)=\text { const }
$$

respectively

$$
\lim _{\sigma \rightarrow \pm \infty} u(\tau, \sigma)=\lim _{\sigma \rightarrow \pm \infty} \bar{u}(\tau, \sigma)=0
$$

then the initial values for the derivatives are determined by eq. (69)

$$
\begin{aligned}
&\left.y_{1}^{\prime}\right|_{\sigma \rightarrow-\infty} \sim-\partial_{z} \bar{u} \bar{C}_{2},\left.y_{2}^{\prime}\right|_{\sigma \rightarrow-\infty} \sim \partial_{z} \bar{u} \bar{C}_{1}, \\
&\left.\bar{y}_{1}^{\prime}\right|_{\sigma \rightarrow-\infty} \sim-\partial_{\bar{z}} u C_{2},\left.\quad \bar{y}_{2}^{\prime}\right|_{\sigma \rightarrow-\infty} \sim \partial_{\bar{z}} u C_{1},
\end{aligned}
$$

so that the solutions of the Gelfand-Dikii equations $y_{k}, \bar{y}_{k}$, and likewise the $A(z), B(z), \bar{A}(\bar{z}), \bar{B}(\bar{z})$, are given uniquely as functions of the physical fields.

These boundary conditions also fix the integration constants $\nu_{0}, \bar{\nu}_{0}$ of (56)

$$
\nu_{0}=-\bar{\nu}_{0}=-\mathrm{i} \ln \left(C_{1} \bar{C}_{1}+C_{2} \bar{C}_{2}\right),
$$

and the conserved $V_{ \pm}, \bar{V}_{ \pm}$are defined as well.

However, in order to calculate Poisson brackets it is not necessary to get $y_{k}(z), \bar{y}_{k}(\bar{z})$ explicitely as functions of $r, t$, respectively $u, \bar{u}$. We only need functional relations among their variations.

\section{Calculation of the Symplectic Structure of the $\mathrm{SL}(2, \mathbb{R}) / \mathrm{U}(1)$ Field Theory}

As a consequence of the solved initial state problem, the Poisson brackets of the (anti-) chiral fields are determined uniquely by those of the physical fields (65, 67). To get the Poisson brackets of the $y_{k}, \bar{y}_{k}$ we shall calculate the variations $\delta y_{k}(z), \delta \bar{y}_{k}(\bar{z})$, e.g., as functions of the variations $\delta u(\tau, \sigma), \delta \bar{u}(\tau, \sigma)$, $\delta \pi_{u}(\tau, \sigma)$ and $\delta \pi_{\bar{u}}(\tau, \sigma)$ by varying the Gelfand-Dikii equations (neglecting the anti-chiral part again)

$$
\delta y_{k}^{\prime \prime}-\left(\partial_{z} V_{-} / V_{-}\right) \delta y_{k}^{\prime}-\gamma^{2} T \delta y_{k}=\delta\left(\partial_{z} V_{-} / V_{-}\right) y_{k}^{\prime}+\gamma^{2} \delta T y_{k},
$$


and the initial state conditions (using again the argument of (73) that also $\delta u, \delta \bar{u}$ are non-zero only in a finite but arbitrary large region)

$$
\begin{aligned}
& \left.\delta y_{k}\right|_{\sigma=-\infty}=\delta C_{k}=0, \\
& \left.\delta y_{1}^{\prime}\right|_{\sigma \rightarrow-\infty}=\left.\delta y_{2}^{\prime}\right|_{\sigma \rightarrow-\infty}=0 .
\end{aligned}
$$

We have to solve, finally, the initial state problem of these inhomogeneous differential equations. For its homogeneous part we take the solutions of the Gelfand-Dikii equations $y_{k}(z)$ and solve the inhomogeneous equation by standard methods with the initial values (77). The result becomes

$$
\begin{aligned}
\delta y_{k}(z)= & \int_{-\infty}^{z} \mathrm{~d} z^{\prime} \Omega\left(z, z^{\prime}\right)\left(\delta\left(\partial V_{-} / V_{-}\right)\left(z^{\prime}\right) y_{k}^{\prime}\left(z^{\prime}\right)+\gamma^{2} \delta T\left(z^{\prime}\right) y_{k}\left(z^{\prime}\right)\right), \\
& \Omega\left(z, z^{\prime}\right) \equiv \frac{y_{1}\left(z^{\prime}\right) y_{2}(z)-y_{2}\left(z^{\prime}\right) y_{1}(z)}{y_{1}\left(z^{\prime}\right) y_{2}^{\prime}\left(z^{\prime}\right)-y_{2}\left(z^{\prime}\right) y_{1}^{\prime}\left(z^{\prime}\right)} .
\end{aligned}
$$

The Poisson brackets

$$
\begin{aligned}
\left\{y_{k}(z), y_{l}\left(z^{\prime}\right)\right\}= & \int_{-\infty}^{z} \mathrm{~d} \tilde{z} \int_{-\infty}^{z^{\prime}} \mathrm{d} \tilde{z}^{\prime} \Omega(z, \tilde{z}) \Omega\left(z^{\prime}, \tilde{z}^{\prime}\right) \times \\
\times & \left(\left\{\left(\partial V_{-} / V_{-}\right)(\tilde{z}),\left(\partial V_{-} / V_{-}\right)\left(\tilde{z}^{\prime}\right)\right\} y_{k}^{\prime}(\tilde{z}) y_{l}^{\prime}\left(\tilde{z}^{\prime}\right)+\right. \\
& +\gamma^{2}\left\{T(\tilde{z}),\left(\partial V_{-} / V_{-}\right)\left(\tilde{z}^{\prime}\right)\right\} y_{k}(\tilde{z}) y_{l}^{\prime}\left(\tilde{z}^{\prime}\right)+ \\
& +\gamma^{2}\left\{\left(\partial V_{-} / V_{-}\right)(\tilde{z}), T\left(\tilde{z}^{\prime}\right)\right\} y_{k}^{\prime}(\tilde{z}) y_{l}\left(\tilde{z}^{\prime}\right)+ \\
& \left.+\gamma^{4}\left\{T(\tilde{z}), T\left(\tilde{z}^{\prime}\right)\right\} y_{k}(\tilde{z}) y_{l}\left(\tilde{z}^{\prime}\right)\right)
\end{aligned}
$$

can now be calculated directly from the canonical brackets of the physical fields (65, 67). The result looks rather simple after integration over $\delta$-functions

$$
\left\{y_{k}(z), y_{l}\left(z^{\prime}\right)\right\}=\frac{\gamma^{2}}{2}\left(y_{k}(z) y_{l}\left(z^{\prime}\right)-y_{l}(z) y_{k}\left(z^{\prime}\right)\right) \epsilon\left(z-z^{\prime}\right) .
$$

$\epsilon(z)$ is the sign function

$$
\epsilon(z) \equiv\left\{\begin{array}{r}
-1 \text { for } z<0 \\
0 \text { for } z=0 \\
1 \text { for } z>0
\end{array}\right.
$$

From this and (60) the non-vanishing Poisson brackets of $A(z)$ and $B(z)$ easily follow as

$$
\begin{aligned}
& \left\{A(z), A\left(z^{\prime}\right)\right\}=\frac{\gamma^{2}}{2}\left(A(z)-A\left(z^{\prime}\right)\right)^{2} \epsilon\left(z-z^{\prime}\right), \\
& \left\{A(z), B\left(z^{\prime}\right)\right\}=\frac{\gamma^{2}}{2}\left(A(z)-A\left(z^{\prime}\right)\right) \epsilon\left(z-z^{\prime}\right) .
\end{aligned}
$$


Our calculations are not finished before we have found a free-field realization of this symplectic structure.

\section{The Canonical Free-Field Transformation}

There are several methods to find relations between $y_{k}(z), \bar{y}_{k}(\bar{z})$ and chiral, respectively anti-chiral components $\phi_{k}(z), \bar{\phi}_{k}(\bar{z})$ of canonical free fields $(k=$ $1,2)$

$$
\psi_{k}(\sigma, \tau)=\phi_{k}(z)+\bar{\phi}_{k}(\bar{z}) .
$$

The most easiest and straight forward approach identifies energy-momentum tensors

$$
T(z)=\left(\partial_{z} \phi_{1}\right)^{2}+\left(\partial_{z} \phi_{2}\right)^{2}=\frac{1}{\gamma^{2}} \frac{y_{1}^{\prime \prime} y_{2}^{\prime}-y_{1}^{\prime} y_{2}^{\prime \prime}}{y_{1} y_{2}^{\prime}-y_{1}^{\prime} y_{2}},
$$

and the same holds for the anti-chiral component.

Here it is appropriate to introduce complex free fields

$$
\psi=\psi_{1}+\mathrm{i} \psi_{2}, \quad \bar{\psi}=\psi_{1}-\mathrm{i} \psi_{2},
$$

which factorize the components of the energy-momentum tensor

$$
T(z)=\partial_{z} \psi \partial_{z} \bar{\psi}, \quad \bar{T}(z)=\partial_{\bar{z}} \psi \partial_{\bar{z}} \bar{\psi} .
$$

Eq. (83) gives a corresponding chiral decomposition of $\psi$ and $\bar{\psi}$

$$
\psi(\sigma, \tau)=\phi(z)+\bar{\chi}(\bar{z}), \quad \bar{\psi}(\sigma, \tau)=\chi(z)+\bar{\phi}(\bar{z}),
$$

with

$$
\begin{aligned}
\phi(z) & =\phi_{1}(z)+\mathrm{i} \phi_{2}(z), & \bar{\phi}(\bar{z}) & =\bar{\phi}_{1}(\bar{z})-\mathrm{i} \bar{\phi}_{2}(\bar{z}), \\
\chi(z) & =\phi_{1}(z)-\mathrm{i} \phi_{2}(z), & \bar{\chi}(\bar{z}) & =\bar{\phi}_{1}(\bar{z})+\mathrm{i} \bar{\phi}_{2}(\bar{z}) .
\end{aligned}
$$

Now we assume that the free fields are local functions of the $y_{k}, \bar{y}_{k}$, which is true for all other quantities of the theory. The most general solution of the ansatz (84) parametrizes the complex free fields as a function of $y_{k}, \bar{y}_{k}$ by eight arbitrary complex constants $\alpha, \beta, \bar{\alpha}, \bar{\beta}, C, \bar{C}, D$ and $\bar{D}$ [5]

$$
\begin{array}{ll}
\phi=\frac{1}{\gamma C}\left(\ln \frac{\alpha y_{1}^{\prime}+\beta y_{2}^{\prime}}{y_{1} y_{2}^{\prime}-y_{1}^{\prime} y_{2}}+D\right), & \chi=\frac{C}{\gamma} \ln \left(\alpha y_{1}+\beta y_{2}\right), \\
\bar{\phi}=\frac{1}{\gamma \bar{C}}\left(\ln \frac{\bar{\alpha} \bar{y}_{1}^{\prime}+\bar{\beta}_{2}^{\prime} \bar{y}_{1}^{\prime} \bar{y}_{2}^{\prime}-\bar{y}_{1}^{\prime} \bar{y}_{2}}{\left.\bar{y}_{1}\right),}\right. & \bar{\chi}=\frac{\bar{C}}{\gamma} \ln \left(\bar{\alpha} \bar{y}_{1}+\bar{\beta} \bar{y}_{2}\right) .
\end{array}
$$


This solution provides, indeed, a canonical free-field transformation, in case, the constants do not depend on physical fields. We find from the Poisson brackets of the $y_{k}, \bar{y}_{k}$ the relations

$$
\begin{aligned}
\left\{\phi(z), \chi\left(z^{\prime}\right)\right\} & =-\frac{1}{2} \epsilon\left(z-z^{\prime}\right), \\
\left\{\bar{\phi}(\bar{z}), \bar{\chi}\left(\bar{z}^{\prime}\right)\right\} & =-\frac{1}{2} \epsilon\left(\bar{z}-\bar{z}^{\prime}\right), \\
\left\{\phi(z), \phi\left(z^{\prime}\right)\right\} & =\left\{\chi(z), \chi\left(z^{\prime}\right)\right\}=0 \\
\left\{\bar{\phi}(\bar{z}), \bar{\phi}\left(\bar{z}^{\prime}\right)\right\} & =\left\{\bar{\chi}(\bar{z}), \bar{\chi}\left(\bar{z}^{\prime}\right)\right\}=0 .
\end{aligned}
$$

But these Poisson brackets follow from (88) if the $\phi_{k}, \bar{\phi}_{k}$ satisfy the canonical free-field Poisson brackets

$$
\begin{aligned}
\left\{\phi_{k}(z), \phi_{l}\left(z^{\prime}\right)\right\} & =-\frac{1}{4} \epsilon\left(z-z^{\prime}\right) \delta_{k l}, \\
\left\{\bar{\phi}_{k}(\bar{z}), \bar{\phi}_{l}\left(\bar{z}^{\prime}\right)\right\} & =-\frac{1}{4} \epsilon\left(\bar{z}-\bar{z}^{\prime}\right) \delta_{k l}, \\
\left\{\phi_{k}(z), \bar{\phi}_{l}(\bar{z})\right\} & =0 .
\end{aligned}
$$

This proves that the solutions of the Gelfand-Dikii equations mediate canonical transformations between physical and free fields.

The many free parameters of (89) can be further restricted [5] by taking into account invariance properties of the energy-momentum tensor, the $\mathrm{GL}(2, \mathbb{C})$ invariance, the chosen boundary conditions, and, finally, by choosing special values for remaining parameters, and the free-field relations (89) simplify to

$$
\begin{array}{rlrl}
\phi & =\frac{1}{\gamma} \ln \frac{y_{1}^{\prime}}{y_{1} y_{2}^{\prime}-y_{1}^{\prime} y_{2}}, & \chi & =\frac{1}{\gamma} \ln y_{1}, \\
\bar{\phi}=\frac{1}{\gamma} \ln \frac{\bar{y}_{1}^{\prime}}{\bar{y}_{1} \bar{y}_{2}^{\prime}-\bar{y}_{1}^{\prime} \bar{y}_{2}}, & \bar{\chi}=\frac{1}{\gamma} \ln \bar{y}_{1} .
\end{array}
$$

Solving these relations with respect to $y_{k}(z)$ and $\bar{y}_{k}(\bar{z})$, we obtain the non-local free-field realizations

$$
\begin{aligned}
& y_{1}(z)=\exp (\gamma \chi(z)) \\
& y_{2}(z)=\exp (\gamma \chi(z))\left(\int_{-\infty}^{z} \mathrm{~d} z^{\prime} \gamma \chi^{\prime}\left(z^{\prime}\right) \exp \left(-\gamma \phi\left(z^{\prime}\right)-\gamma \chi\left(z^{\prime}\right)\right)+\mathrm{e}^{2 \pi \mathrm{i} / 3}\right) \\
& \bar{y}_{1}(\bar{z})=\exp (\gamma \bar{\chi}(\bar{z})), \\
& \bar{y}_{2}(\bar{z})=\exp (\gamma \bar{\chi}(\bar{z}))\left(\int_{+\infty}^{\bar{z}} \mathrm{~d} \bar{z}^{\prime} \gamma \bar{\chi}^{\prime}\left(\bar{z}^{\prime}\right) \exp \left(-\gamma \bar{\phi}\left(\bar{z}^{\prime}\right)-\gamma \bar{\chi}\left(\bar{z}^{\prime}\right)\right)+\mathrm{e}^{2 \pi \mathrm{i} / 3}\right)
\end{aligned}
$$


It is easy to show that the free-field Poisson brackets yield, consistently, the Poisson brackets of the $y_{k}(z), \bar{y}_{k}(\bar{z})$, and we could show that these results also follow from the Gelfand-Dikii equations, in case, their coefficients are expressed in terms of the free fields and the initial state problem is solved anew.

This proves, finally, that the non-local free-field transformations of the physical fields $r, t$, respectively $u, \bar{u}$ are canonical transformations, and we can show they are one to one.

Before collecting these transformations in local Bäcklund transformations, we shall add a remarkable consequence of the symplectic structure. The conserved $V_{ \pm}(z)$ which are related to the $\mathrm{SL}(2, \mathbb{R}) / \mathrm{U}(1)$ coset currents satisfy non-linear Poisson brackets

$$
\begin{aligned}
& \left\{V_{ \pm}(\tau, \sigma), V_{ \pm}\left(\tau, \sigma^{\prime}\right)\right\}=\gamma^{2} V_{ \pm}(\tau, \sigma) V_{ \pm}\left(\tau, \sigma^{\prime}\right) \epsilon\left(\sigma-\sigma^{\prime}\right) \\
& \left\{V_{ \pm}(\tau, \sigma), V_{\mp}\left(\tau, \sigma^{\prime}\right)\right\}=-\gamma^{2} V_{ \pm}(\tau, \sigma) V_{\mp}\left(\tau, \sigma^{\prime}\right) \epsilon\left(\sigma-\sigma^{\prime}\right)+\frac{1}{\gamma^{2}} \delta^{\prime}\left(\sigma-\sigma^{\prime}\right)
\end{aligned}
$$

which provides the Virasoro algebra, and conformal weight one for the $V_{ \pm}$

$$
\left\{T(\tau, \sigma), V_{ \pm}\left(\tau, \sigma^{\prime}\right)\right\}=-\left(\partial_{\sigma^{\prime}} V_{ \pm}\left(\tau, \sigma^{\prime}\right) \delta\left(\sigma-\sigma^{\prime}\right)-V_{ \pm}\left(\tau, \sigma^{\prime}\right) \delta^{\prime}\left(\sigma-\sigma^{\prime}\right)\right) \text {. }
$$

The algebra (94) is characteristic for parafermions [15, 6], which are used in the literature for the discussion of quantum properties of gauged WZNW theories. But our intention here is to emphasize especially the dynamics of the theory governed by the general solution of its equations of motion.

\section{The Bäcklund Transformation of the $\mathrm{SL}(2, \mathbb{R}) / \mathrm{U}(1)$ WZNW Field Theory}

Rewriting the relations (87) in terms of (92)

$$
\begin{aligned}
& \psi(\sigma, \tau)=\frac{1}{\gamma} \ln \frac{y_{1}^{\prime}(z)}{y_{1}(z) y_{2}^{\prime}(z)-y_{1}^{\prime}(z) y_{2}(z)}+\frac{1}{\gamma} \ln \bar{y}_{1}(\bar{z}), \\
& \bar{\psi}(\sigma, \tau)=\frac{1}{\gamma} \ln \frac{\bar{y}_{1}^{\prime}(\bar{z})}{\bar{y}_{1}(\bar{z}) \bar{y}_{2}^{\prime}(\bar{z})-\bar{y}_{1}^{\prime}(\bar{z}) \bar{y}_{2}(\bar{z})}+\frac{1}{\gamma} \ln y_{1}(z)
\end{aligned}
$$


using (62), and eliminating the functions $y_{k}(z), \bar{y}_{k}(\bar{z})$ and their derivatives gives the Bäcklund transformation between the physical and the free fields

$$
\begin{aligned}
& \partial_{z} u=\frac{\gamma}{2} \mathrm{e}^{\gamma \psi}\left(P+\sqrt{P^{2}-4 Q}\right) \partial_{z} \psi, \quad \partial_{\bar{z}} u=\frac{\gamma}{2} \mathrm{e}^{\gamma \psi}\left(P-\sqrt{P^{2}-4 Q}\right) \partial_{\bar{z}} \psi \\
& \partial_{z} \bar{u}=\frac{\gamma}{2} \mathrm{e}^{\gamma \bar{\psi}}\left(P-\sqrt{P^{2}-4 Q}\right) \partial_{z} \bar{\psi}, \quad \partial_{\bar{z}} \bar{u}=\frac{\gamma}{2} \mathrm{e}^{\gamma \bar{\psi}}\left(P+\sqrt{P^{2}-4 Q}\right) \partial_{\bar{z}} \bar{\psi}
\end{aligned}
$$

$P$ and $Q$ are

$P(u, \bar{u}, \psi, \bar{\psi}) \equiv u \mathrm{e}^{-\gamma \psi}+\bar{u} \mathrm{e}^{-\gamma \bar{\psi}}+\mathrm{e}^{-\gamma \psi-\gamma \bar{\psi}}, \quad Q(u, \bar{u}, \psi, \bar{\psi}) \equiv(1+u \bar{u}) \mathrm{e}^{-\gamma \psi-\gamma \bar{\psi}}$.

The integrability conditions for the fields $u$ and $\bar{u}$

$$
\begin{aligned}
0 & =\partial_{\bar{z}} \partial_{z} u-\partial_{z} \partial_{\bar{z}} u=\gamma \mathrm{e}^{\gamma \psi} \sqrt{P^{2}-4 Q} \partial_{z} \partial_{\bar{z}} \psi \\
0 & =\partial_{\bar{z}} \partial_{z} \bar{u}-\partial_{z} \partial_{\bar{z}} \bar{u}=-\gamma \mathrm{e}^{\gamma \bar{\psi}} \sqrt{P^{2}-4 Q} \partial_{z} \partial_{\bar{z}} \bar{\psi}
\end{aligned}
$$

correspond to the free field equations whereas the integrability conditions for the free fields just give the equations of motion for the fields $u$ and $\bar{u}$

$$
\begin{aligned}
& 0=\partial_{\bar{z}} \partial_{z} \psi-\partial_{z} \partial_{\bar{z}} \psi=-\frac{1}{1+u \bar{u}} \sqrt{P^{2}-4 Q}\left(\partial_{z} \partial_{\bar{z}} u-\frac{\bar{u} \partial_{z} u \partial_{\bar{z}} u}{1+u \bar{u}}\right) \\
& 0=\partial_{\bar{z}} \partial_{z} \bar{\psi}-\partial_{z} \partial_{\bar{z}} \bar{\psi}=-\frac{1}{1+u \bar{u}} \sqrt{P^{2}-4 Q}\left(\partial_{z} \partial_{\bar{z}} \bar{u}-\frac{u \partial_{z} \bar{u} \partial_{\bar{z}} \bar{u}}{1+u \bar{u}}\right)
\end{aligned}
$$

This completes the general discussion of the $\mathrm{SL}(2, \mathbb{R}) / \mathrm{U}(1)$ gauged WZNW field theory, and we expect that the canonical free-field transformation prepares the theory for a canonical quantization.

\section{Conclusion}

We have given a proof that any gauged WZNW model is an integrable conformal theory, which was known so far for nilpotent gauging. We have, furthermore, completely solved the $\mathrm{SL}(2, \mathbb{R}) / \mathrm{U}(1)$ theory and calculated its symplectic structure by solving an initial state problem of a Gelfand-Dikii type equation, instead of suggesting this structure only. On this basis we have derived a non-local canonical free-field transformation of the non-linear fields of the theory and we have, finally, collected these transformations in a local Bäcklund transformation.

The calculations were done for a field-theoretic situation with asymptotic boundary conditions. Similar calculations for the periodic case, which include zero modes, will be published elsewhere. 
Although the classical solution of the $\mathrm{SL}(2, \mathbb{R}) / \mathrm{U}(1)$ gauged WZNW model bears strong resemblance to Liouville or Toda theories its quantum structure might be different because its energy-momentum tensor does not have a 'central charge' term classically. But an arising dilaton might compensate for these differences. Thus, an exact quantization of the $\mathrm{SL}(2, \mathbb{R}) / \mathrm{U}(1)$ theory on this basis will be a challange, in particular, with respect to the space-time black hole interpretation of the theory.

\section{Acknowledgement}

We would very much like to thank C.-J. Biebl and Ch. Ford for reading the manuscript and for discussions.

\section{References}

[1] E. Witten, Phys. Rev. D44 (1991) 314.

[2] R. Dijkgraaf, E. Verlinde, H. Verlinde, Nucl. Phys. B371 (1992) 269.

[3] A. A. Tseytlin, Nucl. Phys. B399 (1993) 601.

[4] T.H. Buscher, Phys.Lett. B201 (1988) 466.

[5] U. Müller, thesis 1998, written in German, unpublished.

[6] K. Bardakci, M. Crescimanno, E. Rabinovici, Nucl. Phys. 344 (1990) 344 .

[7] U. Müller and G.Weigt, Phys. Lett. B400 (1997) 21.

[8] J. Balog, L. Feher, P. O'Raifearthaigh, A. Wipf, Phys.Lett. B227 (1989); Annals Phys. 203 (1992) 269.

[9] J.-L. Gervais, M. Saveliev, Phys. Lett. B286 (1992) 271.

[10] P. Goddard, A. Kent, D. Olive, Phys. Lett. B152 (1985) 88.

[11] C.G. Callan, D. Friedan, E.J. Martinec, and M.J. Perry, Nucl. Phys. B262 (1985) 593.

[12] J. Wess, B. Zumino, Phys. Lett. B37 (1986) 78; S. P. Novikov, Sov. Math. Dokl. 37 (1982) 3; E. Witten, Comm. Math. Phys. 92 (1984) 455. 
[13] U. Müller and G.Weigt, in Proceedings of the 30th International Symposium Ahrenshoop, 1996, Buckow, Germany, p. 328.

[14] A. Bilal, Nucl. Phys. B422 (1994) 258.

[15] V.A. Fateev and A.B. Zamolodchikov, Zh.Eksp.Teor.Fiz. 89 (1985)380. 